ARTICLE

DOI: 10.1038/s41467-018-04121-y

\title{
TAK1 activation of alpha-TAT1 and microtubule hyperacetylation control AKT signaling and cell growth
}

Nirav Shah ${ }^{1}$, Sanjay Kumar ${ }^{2}$, Naveed Zaman ${ }^{1}$, Christopher C. Pan ${ }^{3}$, Jeffrey C. Bloodworth ${ }^{4}$, Wei Lei ${ }^{2}$, John M. Streicher ${ }^{2}$, Nadine Hempel ${ }^{5}$, Karthikeyan Mythreye ${ }^{6} \&$ Nam Y. Lee $2,7,8$

Acetylation of microtubules (MT) confers mechanical stability necessary for numerous functions including cell cycle and intracellular transport. Although $\alpha$ TAT1 is a major MT acetyltransferase, how this enzyme is regulated remains much less clear. Here we report TGF- $\beta$-activated kinase 1 (TAK1) as a key activator of $\alpha$ TAT1. TAK1 directly interacts with and phosphorylates $\alpha \mathrm{TAT} 1$ at Ser237 to critically enhance its catalytic activity, as mutating this site to alanine abrogates, whereas a phosphomimetic induces MT hyperacetylation across cell types. Using a custom phospho- $\alpha$ TAT1-Ser237 antibody, we screen various mouse tissues to discover that brain contains some of the highest TAK1-dependent $\alpha$ TAT1 activity, which, accordingly, is diminished rapidly upon intra-cerebral injection of a TAK1 inhibitor. Lastly, we show that TAK1 selectively inhibits AKT to suppress mitogenic and metabolismrelated pathways through MT-based mechanisms in culture and in vivo. Collectively, our findings support a fundamental new role for TGF- $\beta$ signaling in MT-related functions and disease.

\footnotetext{
${ }^{1}$ Division of Pharmacology, College of Pharmacy, The Ohio State University, Columbus, OH, USA. ${ }^{2}$ Deparment of Pharmacology, College of Medicine, University of Arizona, Tucson, AZ, USA. ${ }^{3}$ Department of Pharmacology and Cancer Biology, Duke University, Durham, NC, USA. ${ }^{4}$ Department of Biochemistry and Molecular Biology, Loyola University Chicago, Chicago, IL, USA. ${ }^{5}$ Department of Pharmacology, Penn State University, State College, PA, USA. ${ }^{6}$ Department of Chemistry and Biochemistry, University of South Carolina, Columbia, SC, USA. ${ }^{7}$ Department of Chemistry and Biochemistry, University of Arizona, Tucson, AZ, USA. ${ }^{8}$ The University of Arizona Cancer Center, Tucson, AZ, USA. Correspondence and requests for materials should be addressed to N.Y.L. (email: namlee@email.arizona.edu)
} 
$\mathrm{M}$ icrotubules (MTs) are a fundamental part of the eukaryotic cytoskeletal system that coordinates diverse cellular processes including cell cycle, cell polarity, and intracellular transport ${ }^{1-3}$. As highly dynamic structures comprised $\alpha$ and $\beta$ tubulin heterodimers, MTs continuously polymerize and depolymerize through interactions with various motor proteins and MT-associated proteins (MAPs). In addition, numerous posttranslational modifications (PTMs) have been shown to modulate its overall stability and function ${ }^{4,5}$. Among them, acetylation of a-tubulin at Lys40 is particularly intriguing, as it is the only PTM known to occur on the luminal side yet exerts distinct effects on intracellular transport of vesicles and protein cargo occurring on the outer surface of MTs.

Among the known acetyltransferases, there is mounting evidence that $\alpha$-tubulin acetyltransferase 1 ( $\alpha$ TAT1), a mammalian ortholog of MEC17 found in Caenorhabditis elegans, is considered the major, if not sole, MT-acetylating enzyme in vivo. Indeed, two independently derived aTAT1 knockout mouse models confirm that virtually all cell and tissue types are devoid of a-tubulin acetylation ${ }^{6,7}$. Surprisingly, aTAT1 is not essential for survival during development, although sensory deficits and other higher cognitive functions are likely compromised ${ }^{8}$. Its functional roles are more evident at the cellular level where aTAT1 dysfunction underlies defects in ciliary mechanotransduction, contact inhibition of proliferation, and directional cell migration ${ }^{9,10}$. aTAT1 may also have critical pathophysiologic roles in cancer and many neurodegenerative conditions $7,10,11$. These findings together with previous biophysical structural characterization provide clear fundamental roles for aTAT1 in MT functional modifications. However, aside from a study linking reactive oxygen species (ROS) and AMPK to MT hyperacetylation, there has been very limited knowledge of how this enzyme is catalytically regulated ${ }^{12}$.

Transforming growth factor $\beta$ (TGF- $\beta$ ) is a multifunctional cytokine belonging to a superfamily of more than 30 members $^{13,14}$. The prototype TGF- $\beta 1$ along with many other members signal through the canonical type I receptor T $\beta$ RI (ALK5) to elicit small mothers against decapentaplegic (SMAD) 2/3 transcriptional responses ${ }^{15,16}$. TGF- $\beta$ activation of the SMADindependent pathways occurs primarily through TGF- $\beta$ activated kinase 1 (TAK1), a serine/threonine kinase with essential roles in viability and homeostasis in a variety of cell types and organs ${ }^{17-19}$. TAK1 was initially identified as an effector of TGF- $\beta$-induced p38/JNK activation, although bone morphogenetic proteins, pro-inflammatory molecules such as TNFa and lipopolysaccharides (LPSs), and many others have now been identified as inducers ${ }^{20-22}$. Upon TGF- $\beta$ stimulation, TAK1 phosphorylates the IKK complex and mitogen-activated protein kinase kinases to induce nuclear factor- $\mathrm{kB}$ and mitogen-activated protein kinases such as ERK, p38, and $\mathrm{JNK}^{23}$.

Although MTs mediate the intracellular transport of numerous signaling molecules including SMADs, it is currently unknown whether any of these TGF- $\beta$ effectors directly impact MT functions in real time. This is in stark contrast to a number of studies demonstrating rapid, SMAD-independent mechanisms by which TGF- $\beta$ controls actin cytoskeletal remodeling ${ }^{24,25}$. In the present study, we establish a fundamental new role for TGF- $\beta$ signaling in MT dynamics and function. Through mass spectrometry and proteomics screening, we identified aTAT1 as a novel binding partner for TAK1. Here we describe the mechanisms by which TGF- $\beta$ exerts rapid, non-transcriptional effects on MT acetylation and downstream cellular functions through TAK1.

\section{Results}

TAK1 directly binds to aTAT1 to enhance MT acetylation. In search of new binding partners for the major TGF- $\beta$ effectors present in most cell types, we performed a mass spectrometrybased proteomics on SMADs 1, 2, and TAK1. Interestingly, among the numerous hits from the interactome screening, we noted aTAT1 as a potential binding partner for TAK1 with new functional implications (Supplementary Fig. 1). Subsequent biochemical studies confirmed their novel interaction as aTAT1 was pulled down upon TAK1 immunoprecipitation (Fig. 1a). A kinase-dead version of TAK1 (K63W point mutant) also bound efficiently with aTAT1, suggesting that the TAK1 catalytic activity is dispensable at least for their interaction (Fig. 1a), whereas parallel experiments showed that none of the SMAD proteins associated with this MT enzyme. To better understand the molecular basis of this interaction, pharmacologic inhibitors SB43152 and 5Z-7-Oxozeaenol were used to inhibit the kinases of T $\beta R I$ and TAK1, respectively. Here, the TAK1/aTAT1 interaction was greatly enhanced upon inhibiting the activity of TAK1 but not T $\beta$ RI kinase, indicating that aTAT1 may be a catalytic substrate for TAK1 (Fig. 1b). Notably, this interaction was also evident at the endogenous level where the two proteins readily colocalized and co-immunoprecipitated, but was further enhanced upon TGF- $\beta$ stimulation (Fig. 1c, d). Lastly, to test whether their interaction is direct, binding studies were performed using recombinant purified proteins from the baculovirus/insect cell system (Fig. 1e and Supplementary Fig. 4). Here, purified biotinlabeled TAK1 was immobilized on streptavidin matrix and then co-incubated with purified aTAT1. Consistent with the coimmunoprecipitation data, aTAT1 was efficiently pulled down by biotin-labeled TAK1 to confirm their direct interaction (Fig. 1e).

Whether TGF- $\beta$ controls the dynamics or PTMs of MTs in a rapid, non-transcriptional manner has never been reported. However, given our data demonstrating a direct interaction between TAK1 and aTAT1, the primary MT-acetylating enzyme in vivo, we explored the potential for TGF- $\beta$-induced TAK1 in directly modifying MT acetylation across cell types. Here, TGF- $\beta$ caused a rapid increase in MT acetylation as observed by immunofluorescence, while the overall MT architecture remained constant (Fig. 1f, graphs). Accordingly, biochemical analysis showed rapid kinetics where TGF- $\beta$ treatment markedly increased $\alpha$-tubulin acetylation within $15 \mathrm{~min}$ and persisted up to $2 \mathrm{~h}$, while total tubulin levels were unchanged (Fig. 1g). Next, as TGF- $\beta$ signaling is mediated by the canonical T $\beta R I / S m a d 2 / 3$ and TAK1 pathways, cells were briefly treated with SB43152 or 5Z-7-Oxozeaenol and found that TAK1 inhibition reduced, whereas T $\beta R I$ inhibition moderately enhanced MT acetylation, suggesting that TGF- $\beta$ likely causes MT acetylation through TAK1 signaling (Fig. 1h, graphs).

To test whether TAK1 enhances acetylation directly or indirectly through its downstream effectors such as p38 and JNK, cells were preincubated with inhibitors of TAK1, p38 and JNK before TGF- $\beta$ stimulation. Here, an almost twofold increase in acetylation was observed upon TGF- $\beta$ treatment in control, while TAK1 inhibition fully abrogated the effects of TGF- $\beta$ (Fig. 1i, graph). However, blocking p38 or JNK modestly increased basal MT acetylation independent of ligand treatment (Fig. 1i, graph), again supporting the likely direct role of TAK1 in MT acetylation. To further test this hypothesis, TAK1 stable knockdown cell lines (shTAK1) were generated to observe more than $70 \%$ reduction in basal MT acetylation over control cells as assessed by biochemical and immunofluorescence analyses (Fig. 2a, b, graph). Again, total tubulin levels and the overall MT morphology remained constant upon TAK1 depletion, indicating that TAK1 selectively targets MT acetylation (Fig. 2b, graphs).

Although the early events of TGF- $\beta$-induced TAK1 activation requires TAK1 to associate with T $\beta R I$, the T $\beta R I$ kinase activity is surprisingly dispensable unlike the canonical Smad pathways that 
a

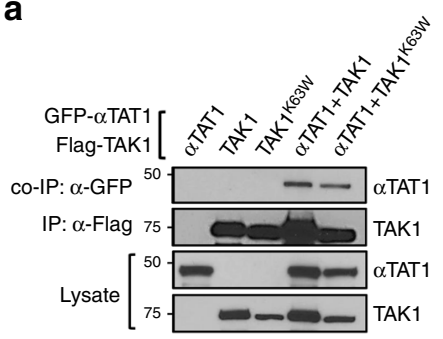

d

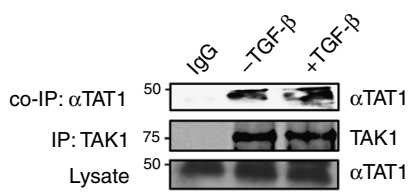

g

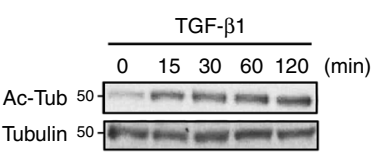

h

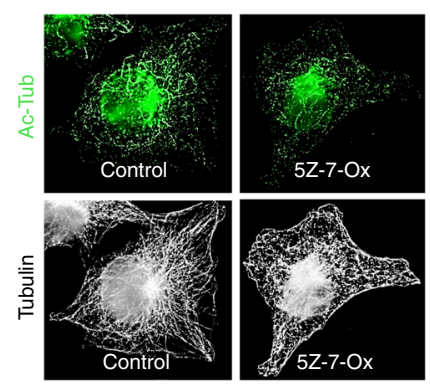

b

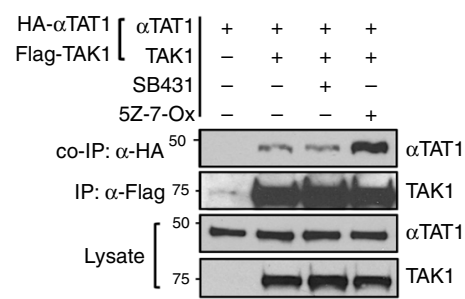

C

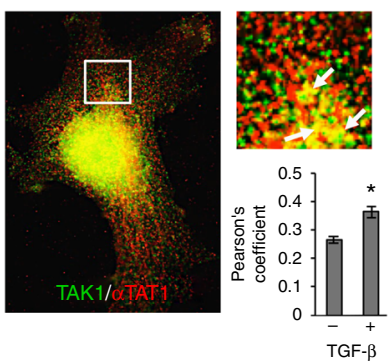

e

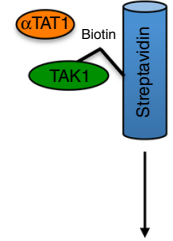

Biotinylation: Immobilized TAK1 75

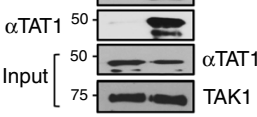

f
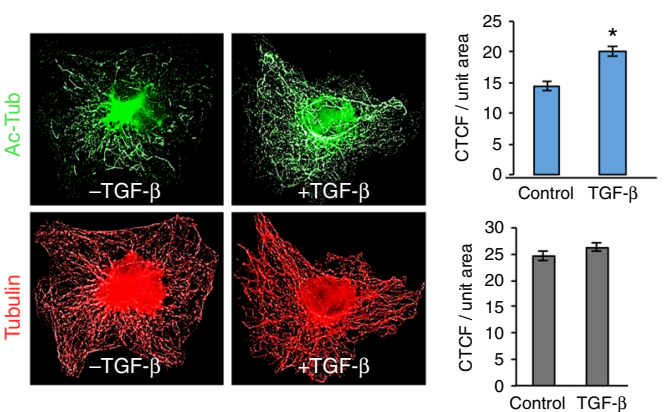

i
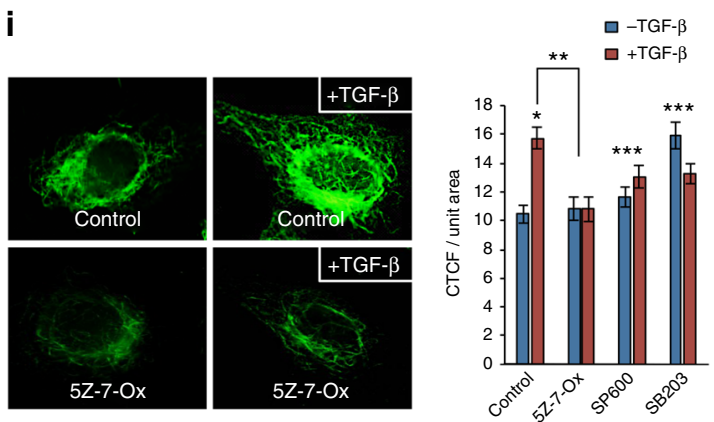

Fig. 1 TAK1 directly binds $\alpha$ TAT1 to promote MT acetylation. a COS-7 cells expressing HA- $\alpha$ TAT1 WT, Flag-TAK1 WT, or Flag-TAK1 kinase-dead (K63W) were immunoprecipitated with Flag antibody. b COS-7 cells expressing HA- $\mathrm{ATAT1}$ WT with or without Flag-TAK1 WT were treated with SB431542 $(30 \mu \mathrm{M})$ or $(5 \mathrm{Z})-7-O x o z e a e n o l(20 \mu \mathrm{M})$ for 30 min before immunoprecipitation with Flag antibody. c COS-7 cells were treated with TGF- $\beta 1$ (200 pM) for 30 min before fixing and then imaged for endogenous $\alpha$ TAT1 (C6ORF) and TAK1 immunofluorescence staining. At least 15 cells per condition (3 ROIs per cell) were quantified using Image J plugin JACoP to determine Pearson's correlation coefficient. Error bars represent SEM from three independent experiments $\left({ }^{*} p=0.0001\right)$. d COS-7 cells were treated with TGF- $\beta 1$ (200 pM) for 30 min and the lysates were immunoprecipitated with either IgG control or $\alpha$ TAT1 (C6ORF) antibody. e Purified $\alpha$ TAT1 and TAK1 were incubated with biotinylated TAK1 immobilized to streptavidin agarose. As a negative control, $\alpha$ TAT1 and non-biotinylated TAK1 were incubated in streptavidin agarose. $\mathbf{f}$ COS-7 cells were treated with TGF- $\beta 1$ (200 pM) for 30 min before fixation, then imaged for acetyl-tubulin (top) and total tubulin (bottom) immunofluorescence staining. The images were quantified for corrected total cell fluorescence (CTCF) per unit area using Image J. Quantification was based on data collected from 50 cells per condition. The images are representative of three independent experiments, $n=3$. Error bars represent SEM and type $2 t$-test results show relative to control: ${ }^{*} p<0.05$. $\mathbf{g}$ Western blot analysis of acetyl and total tubulin post TGF- $\beta 1(200 \mathrm{pM})$ treatment for $0,15,30,60$, and 120 min in COS-7 cells. $\mathbf{h}$ COS-7 cells were treated with (5Z)-7-Oxozeaenol $(20 \mu \mathrm{M})$ or SB431542 $(30 \mu \mathrm{M})$ for 30 min before fixation, then imaged for Acetyl-tubulin (top) and total tubulin (bottom three) immunofluorescence staining. At least 50 cells per condition were quantified for CTCF using Image J. The images are representative of three independent experiments, $n=3$. Error bars represent SEM and type $2 t$-test results show relative to control: ${ }^{*} p<0.05$. i HeLa cells were pre-treated with (5Z)-7Oxozeaenol $(20 \mu \mathrm{M})$, SP600125 $(10 \mu \mathrm{M})$, or SB203580 $(20 \mu \mathrm{M})$ for $30 \mathrm{~min}$ and then subjected to TGF- $\beta 1(200 \mathrm{pM})$ for 30 min before fixation. The images represent Acetyl-tubulin immunofluorescence staining and were quantified for CTCF using Image J. Quantification was based on data collected from at least 30 cells per condition. The images are representative of three independent experiments, $n=3$. Error bars represent SEM. Relative to control: ${ }^{\star} p<0.05 ;{ }^{\star \star} p<0.05 ;{ }^{\star \star \star} \mathrm{NS}$

strictly require the receptor serine/threonine kinase to phosphorylate $\operatorname{Smad} 2 / 3^{19}$. Therefore, we hypothesized that the enhanced MT acetylation previously observed upon SB43152 treatment (Fig. 1h) was largely due to greater access of TAK1 to TGF- $\beta$ bound T $\beta R I$ than Smad2/3. Indeed, whereas MT acetylation was elevated upon T $\beta$ RI kinase inhibition as previously observed in control cells (Fig. 1h), shTAK1 cells displayed strikingly reduced acetylation at basal state, but more importantly, failed to respond upon SB43152 treatment (Fig. 2c, graph). Expression of T $\beta R I$ kinase-dead (ALK5 ${ }^{\mathrm{K} 232 \mathrm{R}}$ ) also yielded similar results where TAK1 still induced MT acetylation in response to TGF- $\beta$ while failing to activate Smad2 (Fig. 2d). Further substantiating this 


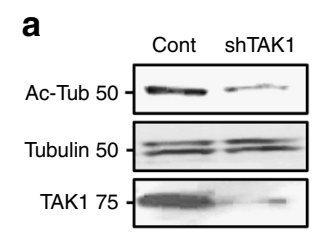

b
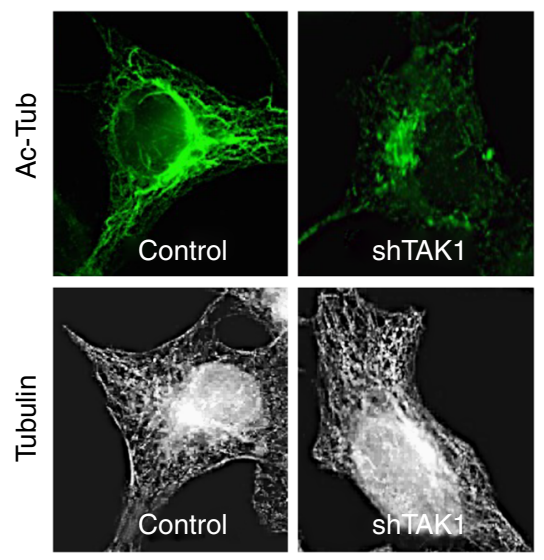
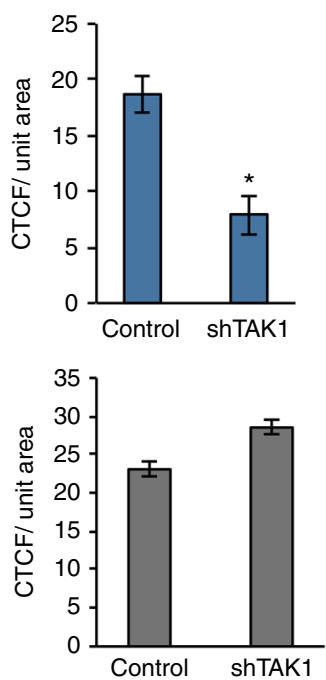

C
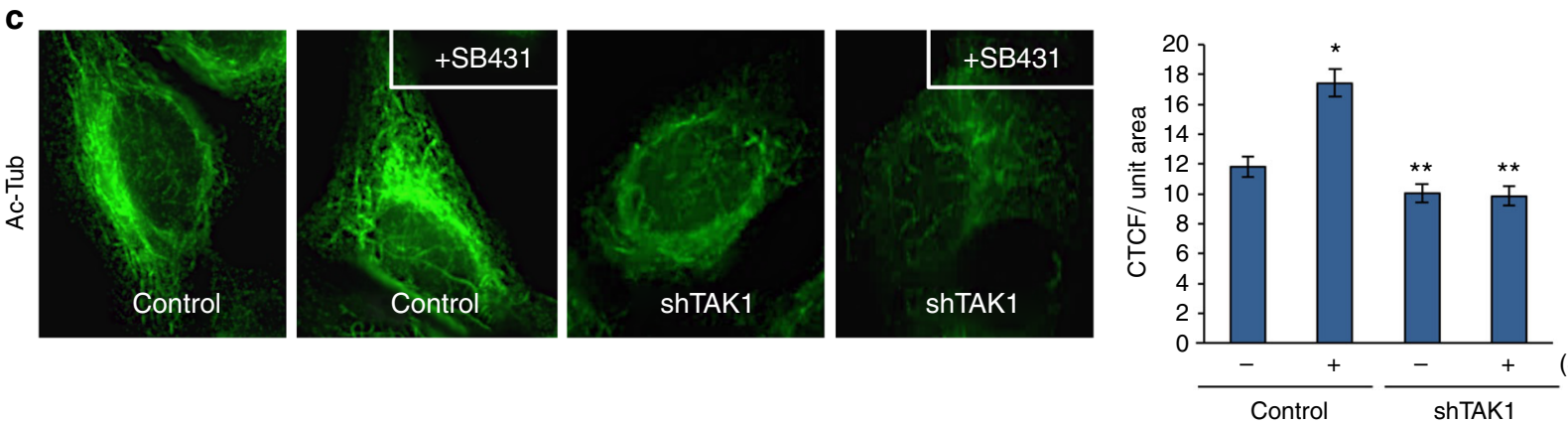

(SB431)

d

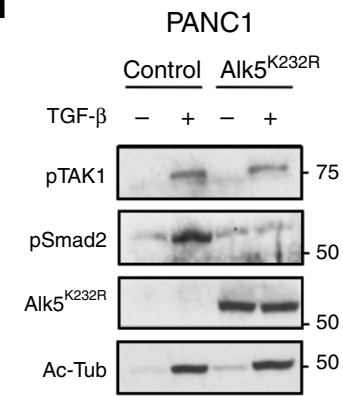

e

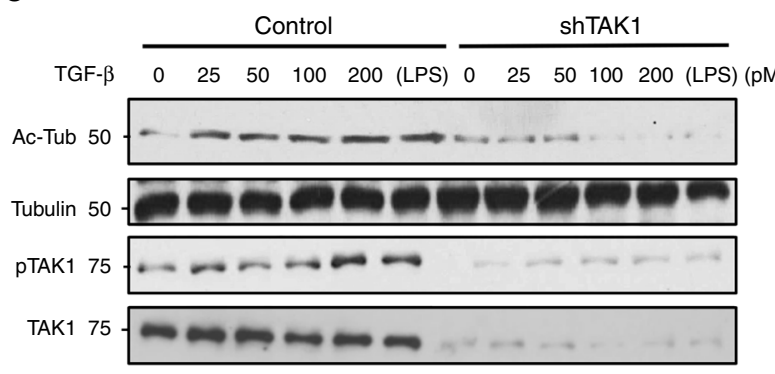

Fig. 2 TAK1 depletion abrogates TGF- $\beta$-induced MT acetylation. a Western blot analysis of acetyl-tubulin, total tubulin, and total TAK1 in control HeLa and sh-TAK1 stable cells. b Control HeLa and sh-TAK1 stable cells imaged for acetyl tubulin and total tubulin immunofluorescence staining. The images were quantified for CTCF using Image J. At least 30 cells per condition were quantified, the images are representative of four independent experiments, $n$ $=4$. Error bars represent SEM and type $2 t$-test show relative to control: ${ }^{*} p<0.001$. $\mathbf{c}$ Control HeLa and sh-TAK1 stable cells were treated with SB431542 $(30 \mu \mathrm{M})$ for $30 \mathrm{~min}$ before fixing and then imaged for acetyl tubulin immunofluorescence staining. The images were quantified for CTCF using Image J. At least 30 cells per condition were quantified. The images are representative of three independent experiments, $n=3$, error bars represent SEM and type $2 t$ test show relative to control: ${ }^{\star} p<0.05,{ }^{\star \star} p<0.01$. d Panc1 cells were transiently transfected with PCDNA control vector or myc-ALK5-K232R construct followed by treatment with TGF- $\beta 1(200 \mathrm{pM} ; 1 \mathrm{~h})$ before western blot analysis of phospho-TAK1, phospho-Smad2, ALK5-K232R, and acetyl tubulin. e Control HeLa and sh-TAK1 stable cells were treated with increasing concentrations of TGF- $\beta 1(0,25,50,100$, and 200 pM) and $10 \mathrm{ng} / \mathrm{ml} \mathrm{LPS}$ before western blot analysis of Acetyl-tubulin, total tubulin, phospho-TAK1, and total TAK1

finding, a TGF- $\beta$ dose-response study revealed a steady, concentration-dependent increase in both tubulin acetylation and TAK1 phosphorylation in control but not in shTAK1 cells (Fig. 2e). Similarly, we observed that LPS, a known TAK1 inducer, enhanced acetylation in a TAK1-dependent manner (Fig. 2e; lane 6). Taken together, these data strongly supported TAK1 as the principal mediator of TGF- $\beta$-induced MT acetylation.

Next, we sought to identify the critical structural determinants of the TAK1/aTAT1 complex by first testing a TAK1 C-terminal truncation mutant that retains the kinase domain ( $\mathrm{TAK} 1^{\Delta \mathrm{CT}}$ ) (Fig. 3a). Here, the immunoprecipitation of the full-length TAK1 strongly associated with aTAT1, whereas TAK1 ${ }^{\Delta \mathrm{CT}}$ failed to interact (Fig. 3b). The reciprocal experiment in which aTAT1 was immunoprecipitated also yielded a consistent pattern, thus indicating that the TAK1 C-terminal domain is essential for their interaction (Fig. 3c). Notably, immunofluorescence data showed that TAK1-WT expressing cells conferred at least three-fold increase in MT acetylation compared with both the kinase-dead mutant $\mathrm{TAK}^{\mathrm{K} 63 \mathrm{~W}_{-}}$and $\mathrm{TAK} 1^{\Delta \mathrm{CT}}$-expressing cells, which 


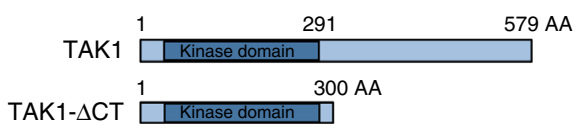

d

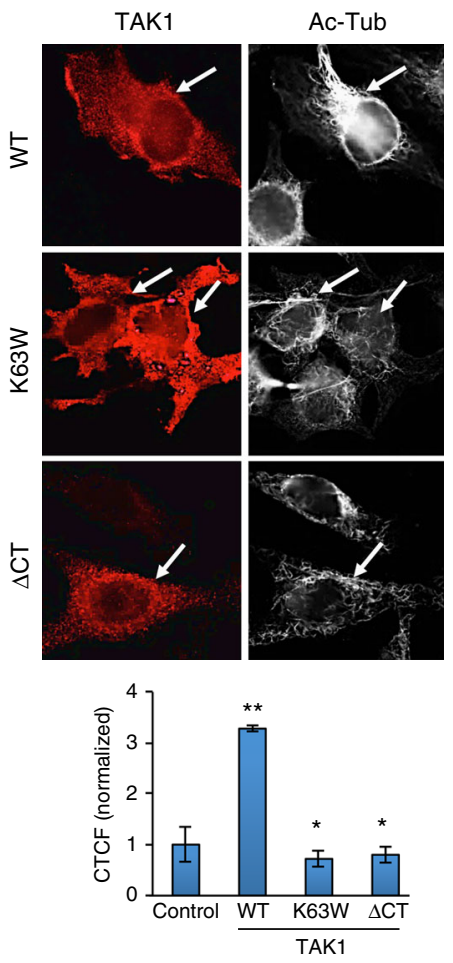

b

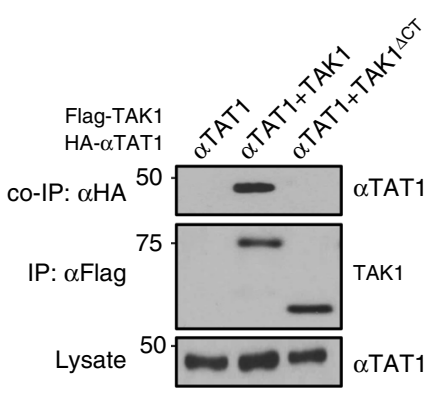

e

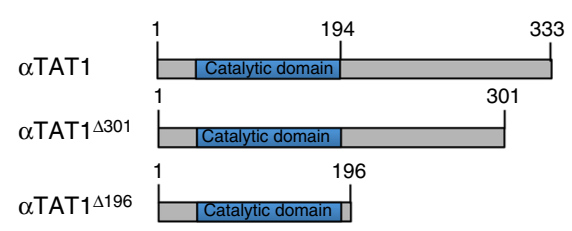

g

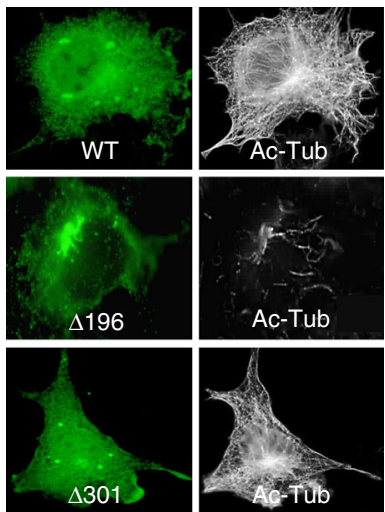

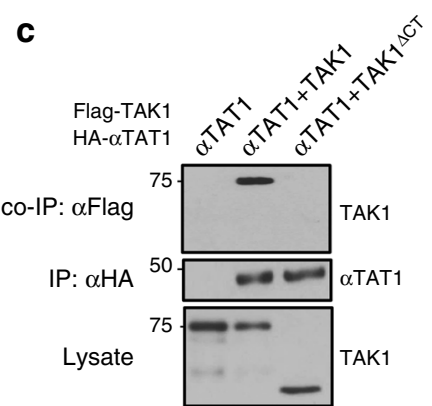

f
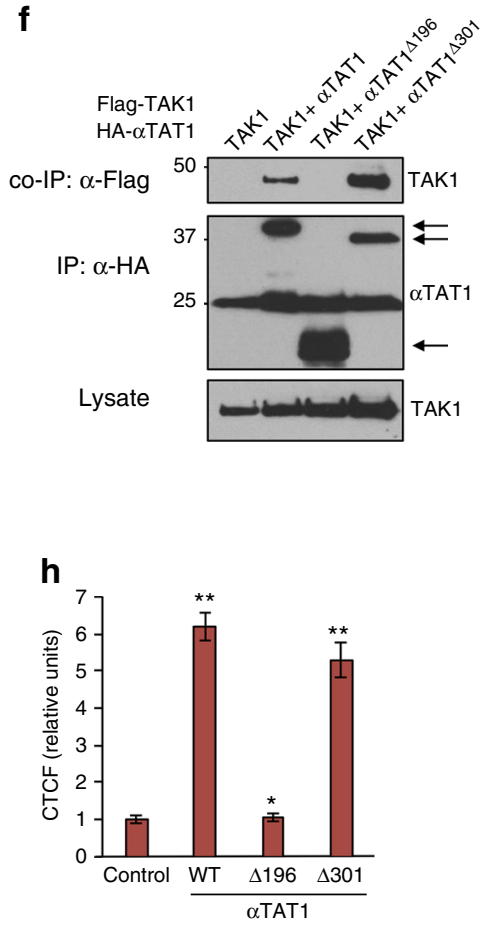

Fig. 3 Structural determinants of TAK1 required for $\alpha$ TAT1 binding. a Schematic representation of TAK1 WT and C-terminal truncated mutant TAK1$\triangle$ CT. b COS-7 cells expressing HA- $\alpha$ TAT1 WT, Flag-TAK1, or Flag- TAK1- $\triangle C T$ were immunoprecipitated with Flag antibody. c COS-7 cells expressing HA-

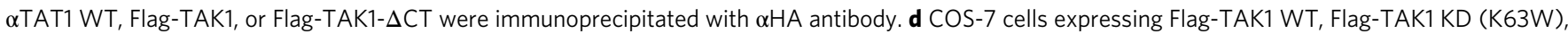
and Flag-TAK1- $\triangle C$ T were fixed and imaged for Flag (left panel) and Acetyl-tubulin (right panel) immunofluorescence co-staining. CTCF was quantified using Image J, at least 30 cells were quantified per condition. The images are representative of three independent experiments, $n=3$. Error bars represent SEM and type $2 t$-test analysis show relative to control: ${ }^{*}$ not significant; ${ }^{* *} p<0.001$. e Schematic representation of $\alpha$ TAT1-WT and truncation mutants $\alpha$ TAT1- $\Delta 301, \alpha T A T 1-\Delta 196$. f COS-7 cells expressing Flag-TAK1 WT, HA- $\alpha$ TAT1 WT, HA- $\alpha$ TAT1- $\Delta 196$, or HA- $\alpha$ TAT1- $\Delta 301$ were immunoprecipitated with $\alpha-\mathrm{HA}$ antibody. $\mathbf{g}$, h COS-7 cells expressing HA- $\alpha$ TAT1 WT, HA- $\alpha$ TAT1- $\Delta 196$, or HA- $\alpha$ TAT1- $\Delta 301$ were fixed and imaged for $\alpha-\mathrm{HA}$ (left panel) and Acetyltubulin (right panel) immunofluorescence co-staining. CTCF was quantified using Image J, at least 30 cells were quantified per condition. The images are representative of three independent experiments, $n=3$. Error bars represent SEM and type $2 t$-test analysis show relative to control: ${ }^{*}$ not significant; ${ }^{\star \star} p<0.001$

remained at basal state similar to the surrounding nontransfected cells (Fig. 3d, arrows and graph).

To define the aTAT1 structural elements, we generated Cterminal deletions that truncate either the last 33 amino acids $\left(\right.$ aTAT $\left.^{\Delta 301}\right)$ or the last 137 residues that still retains the acetyltransferase catalytic domain ( $\mathrm{aTAT}^{\Delta 196}$ ) (Fig. 3e). Here, both the full-length and aTAT1 $1^{\Delta 301}$ interacted efficiently with TAK1, whereas aTAT1 ${ }^{\Delta 196}$ did not, indicating that the aTAT1 Cterminal region is not only critical for the interaction with TAK1 (Fig. 3f) but also required for robust MT acetylation such as the wild type (WT) (Fig. 3g, h).

TAK1 phosphorylates aTAT1 to enhance its catalytic activity. Based on these findings, we tested whether TAK1 mediates MT acetylation either by promoting aTAT1 trafficking/recruitment to the MTs or through direct phosphorylation-induced enzyme activation. To address the former possibility, we assessed the endogenous aTAT1 co-localization with MTs in control and shTAK1 HeLa cells (Supplementary Fig. 2). Here, aTAT1 colocalized within MTs efficiently independent of TAK1 depletion or TGF- $\beta$ stimulation (Supplementary Fig. 2, graph), thereby suggesting that TAK1 does not help recruit aTAT1 to MTs. This finding prompted us to focus on the C-terminal region of aTAT1 as a potential phosphorylation target, as it was deemed critical for its interaction with TAK1 and MT acetylation (Fig. 4a). Initial attempts to identify novel TAK1-induced phosphorylation sites through mass spectrometry proved unsuccessful due to a combination of insufficient sequence coverage and inability to differentiate adjoining serine phosphorylation sites (e.g., Ser236 and Ser237). Further hindering our efforts was the fact that TAK1 phosphosubstrates lack a conserved phosphorylation motif; 
a

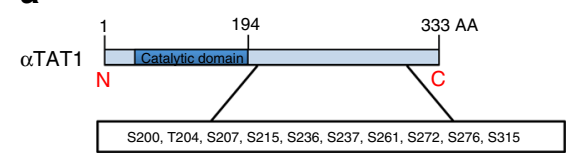

b

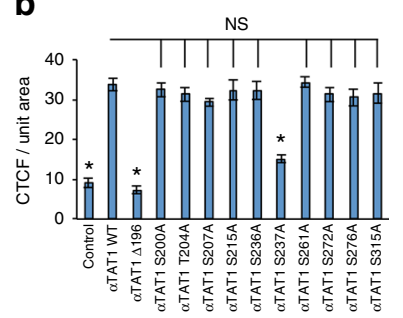

d

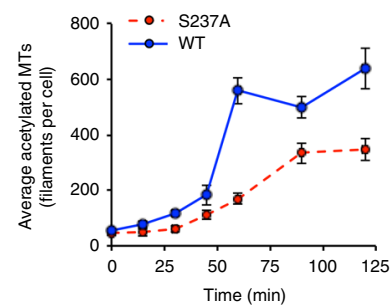

C
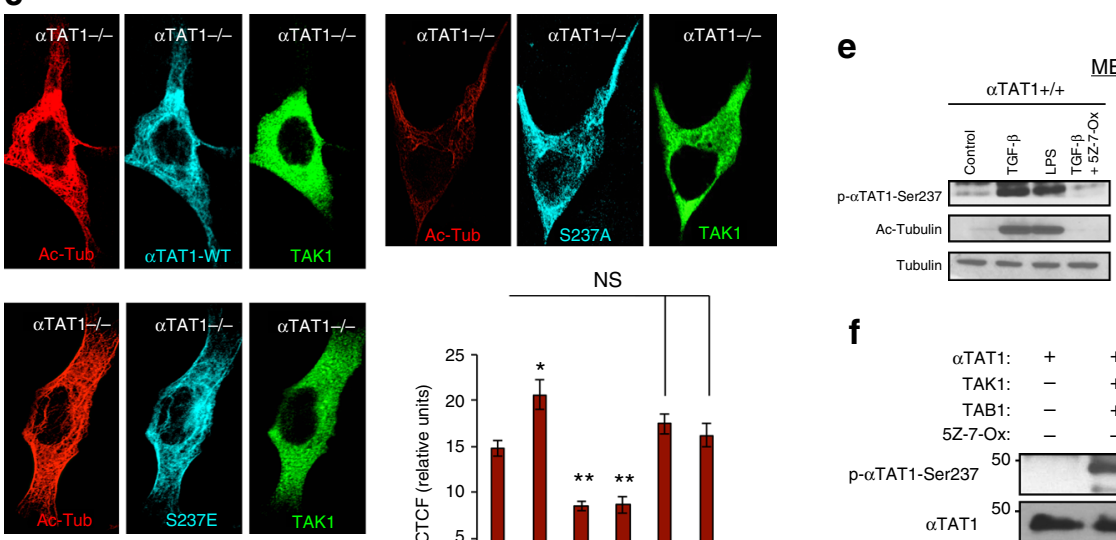

MEFs
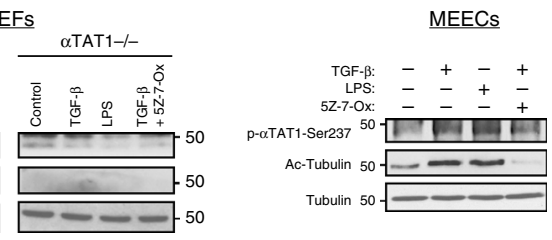

$\mathbf{f}$

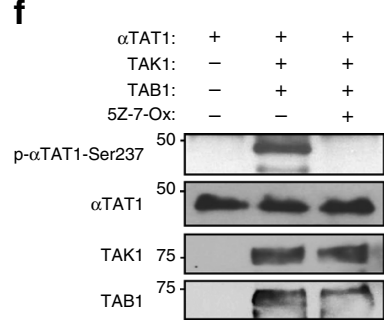

g

$\alpha$ TAT1: WT WT S237A TAK1/TAB1:

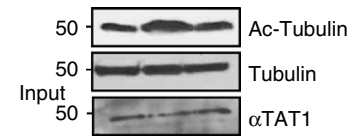

Fig. $4 \alpha$ TAT1 catalytic activation requires direct phosphorylation at Ser237 by TAK1. a Schematic representation of $\alpha$ TAT1 serine/threonine to alanine single point mutants. b COS-7 cells expressing $\alpha \mathrm{TAT1}$ WT or the serine/threonine to alanine single point mutants were fixed and imaged for $\alpha \mathrm{HA}$ and Acetyl-tubulin. CTCF was quantified using Image J, at least 25 cells were quantified per condition. The images are representative of three independent experiments, $n=3$. Error bars represent SEM and type $2 t$-test analysis show relative to $\alpha$ TAT1 WT: ${ }^{*} p<0.001$. c $\alpha$ TAT1 ${ }^{-/-}$MEFs expressing TAK1 WT or HA- $\alpha$ TAT1 WT, HA- $\alpha$ TAT1-S237A, and HA- $\alpha$ TAT1-S237E were fixed and imaged for Acetyl-tubulin (red), $\alpha$ HA (pseudo color cyan), and TAK1 (green) immunofluorescence co-staining. CTCF per unit area was quantified using Image J, at least 30 cells were quantified per condition. Error bars represent SEM and type $2 t$-test analysis show relative to $\alpha$ TAT1 WT: ${ }^{*} p<0.001$; ${ }^{\star \star} p<0.0001$. $\mathbf{d}$ COS-7cells expressing HA- $\alpha$ TAT1 WT or HA- $\alpha$ TAT1-S237A were subjected to nocodazole $(100 \mathrm{nM})$ for $1 \mathrm{~h}$, washed thoroughly with PBS, and then immediately fixed after $0,15,30,45,60,90$, and 120 min. The fixed cells were then imaged for $\alpha \mathrm{HA}$ and Acetyl tubulin immunofluorescence co-staining. The images were quantified by skeletonizing the acetyl tubulin staining using Image $J$ and skeletons analyzed for length and branching of acetyl tubulin filaments. e $\alpha T A T 1^{+/+}$MEFs, $\alpha T A T 1^{-/-}$MEFs, and mouse embryonic endothelial cells were treated with TGF- $\beta(200 \mathrm{pM})$, LPS $(10 \mathrm{ng} / \mathrm{ml})$ for $30 \mathrm{~min}$, or pre-treated with 5Z-7-oxozeaenol $(20 \mu \mathrm{M})$ for $15 \mathrm{~min}$ followed by TGF- $\beta$ stimulation. $\mathbf{f}$ In vitro kinase assay performed using purified $\alpha$ TAT1, TAK1, and TAB1 were incubated with and without treatment with 5Z-7-oxozeaenol $(20 \mu \mathrm{M}) . \mathbf{g}$ In vitro acetylation assay performed using purified tubulin, $\alpha$ TAT1, $\alpha$ TAT1-S237A, TAK1, and TAB1

hence, we systematically mutated every serine and threonine residue within this C-terminal region (residues 200-315) to alanine via site-directed mutagenesis and functionally screened for their MT-acetylating capacity (Fig. 4b). Relative to control at basal state, the WT aTAT1-expressing cells yielded greater than threefold enhancement, whereas aTAT $1^{\Delta 196}$-expressing cells had no effect similar to control as expected (Fig. 4b). However, among the ten additional aTAT1 point mutants tested, all yielded remarkably similar levels of MT acetylation relative to WT with the exception of $\alpha \mathrm{TAT} 1^{\text {S237A }}$ (Fig. $4 \mathrm{~b}$ ).

Hence, to further interrogate the functional role of this phosphorylation site, phosphomimetic constructs (Asp D and Glu E) were generated and each expressed in aTAT1-knockout $\left(\mathrm{aTAT}^{-/-}\right)$mouse embryonic fibroblasts (MEFs). Whereas aTAT1 $^{-/-}$MEFs were devoid of MT acetylation as expected (Supplementary Fig. 3A), the ectopic expression of the WT and those of Ser237D and Ser237E mutants produced robust MT acetylation, whereas Ser237A displayed significantly impaired catalytic activity in both aTAT1 $^{-/-} \mathrm{MEF}$ and COS-7 cells (Fig. 4c, graph). Notably, co-expressing TAK1 led to greater acetylation in WT but not Ser237E or the Ser237A mutant, suggesting that TAK1 acts specifically through this phosphorylation site (Fig. 4c, graph). Similar effects were observed in other cell types including COS-7 cells wherein overexpression of WT and S237D and E, but not S237A, dramatically enhanced MT acetylation over basal non-transfected cells (Supplementary Fig. 3B, graph). Furthermore, we observed that TGF- $\beta$ stimulation enhanced, whereas 5Z-7-Oxozeaenol attenuated MT acetylation in WT aTAT1-expressing cells, while those expressing aTAT1 ${ }^{\text {S237A }}$ failed to respond to either TGF- $\beta$ or $5 Z-7-$ Oxozeaenol (Supplementary Fig. 3C).

However, to exclude the possibility of adaptive mechanisms contributing to MT acetylation, we measured the kinetics of the WT or aTAT1 ${ }^{\text {S237A }}$ upon brief nocodazole treatment to synchronize and abolish MT acetylation (Fig. 4d). Here, subsequent washout allowed for the visualization of steadily growing MTs and their progressive acetylation at various time points. During the $2 \mathrm{~h}$ time course, maximum MT acetylation levels were achieved and then plateued within $1 \mathrm{~h}$ in the WT, whereas aTAT1 ${ }^{\text {S237A }}$ lagged far behind at the $1 \mathrm{~h}$ mark and beyond, reaching roughly $50 \%$ capacity of the WT (Fig. $4 \mathrm{~d}$ ). Hence, these findings strongly supported the TAK1 phosphorylation of aTAT1 on site Ser237 as a key mechanism of MT acetylation.

In order to prove aTAT1-Ser237 as a direct phosphorylation site targeted by TAK1, we raised a custom phospho-specific antibody. However, upon careful testing the antibody was determined to be incompatible with western blotting yet suitable 
for enzyme-linked immunosorbent assays and immunoprecipitation. Thus, we first assessed whether endogenous $\alpha$ TAT1 becomes phosphorylated at Ser237 upon stimulation with either TGF- $\beta$ or LPS followed by immunoprecipitation with the phosphoantibody, and then immunoblotted with pan-aTAT1 antibody. In both MEFs and a mouse endothelial cell line, both TGF- $\beta$ and LPS induced MT acetylation as expected and, more importantly, was accompanied by the detection of phosphorylated aTAT1-Ser237 (Fig. 4e). To further demonstrate that these were TAK1-directed effects, cells were pre-incubated with TAK1 inhibitor before TGF$\beta$ stimulation and found that aTAT1-Ser237 phosphorylation was abrogated along with MT acetylation (Fig. 4e).

To provide definitive evidence of direct TAK1-induced effects on aTAT1-Ser237 phosphorylation, we turned to recombinant purified proteins in a cell-free system. In most cellular contexts, TAK1 associates with TAK1-binding protein (TAB1) upon TGF$\beta$ stimulation to activate the serine/threonine kinase. Although in our co-precipitation studies TAB1 proved to be dispensable for the TAK1/aTAT1 interaction, we generated purified TAK1, $\mathrm{TAB} 1$, and aTAT1 proteins, and performed an in vitro kinase assay in the presence or absence of 5Z-7-Ox preincubation. Here we observed a robust aTAT1-Ser237 phosphorylation with the addition of the TAK1/TAB1 complex, whereas the same complex failed to phosphorylate aTAT1-Ser237 in the presence of 5Z-7Ox (Fig. 4f). Likewise, the recombinant TAK1/TAB1 complex was capable of further acetylation of purified $\alpha$-tubulin in the presence of WT but not aTAT1-Ser237 (Fig. 4g). Collectively, these results strongly support aTAT1 as a novel TAK1 substrate requiring Ser237 phosphorylation for enhancement of aTAT1 catalytic activity.

aTAT1 regulates cell growth and maintenance by AKT inactivation. Although aTAT1 can suppress cell growth through contact inhibition $^{26}$, still a crucial question is whether it acts through MT stabilization, thereby impairing such process as mitotic spindle assembly, or through regulation of intracellular transport of key intracellular signaling molecules. To test this and understand how TGF- $\beta$ contributes to this process, we focused on the role of TAK1 phosphorylation of aTAT1 and MT acetylation in cell growth. As previously reported, aTAT1 ${ }^{-/-}$MEFs proliferated more rapidly over the course of $48 \mathrm{~h}$ than control cells, a trend that was reversed upon rescue expression of the aTAT1 WT (Fig. 5a; 24-48 h). To test whether aTAT1 inhibits cell growth mainly through MT acetylation, and further dissect the role of aTAT1-Ser237 phosphorylation, aTAT1 ${ }^{-/-}$MEFs were transfected with the WT, Ser237A, and Ser237E (Fig. 5b). Here, both the $\mathrm{TTAT}^{-/-}$MEFs and Ser237A-expressing cells had greater rate of proliferation than the WT or the phosphomimetic, suggesting that Ser237 phosphorylation critically regulates cell growth (Fig. 5b). This growth pattern was similarly evidenced in

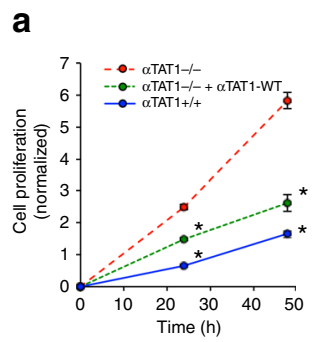

d

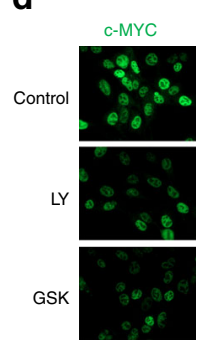

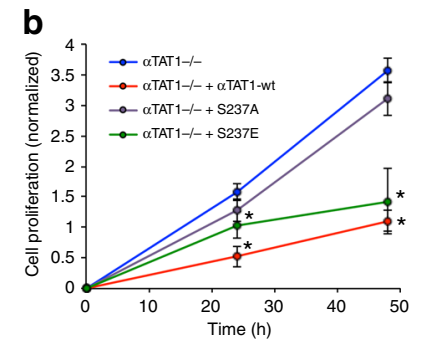

e
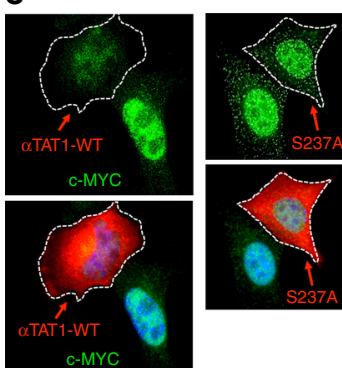

C
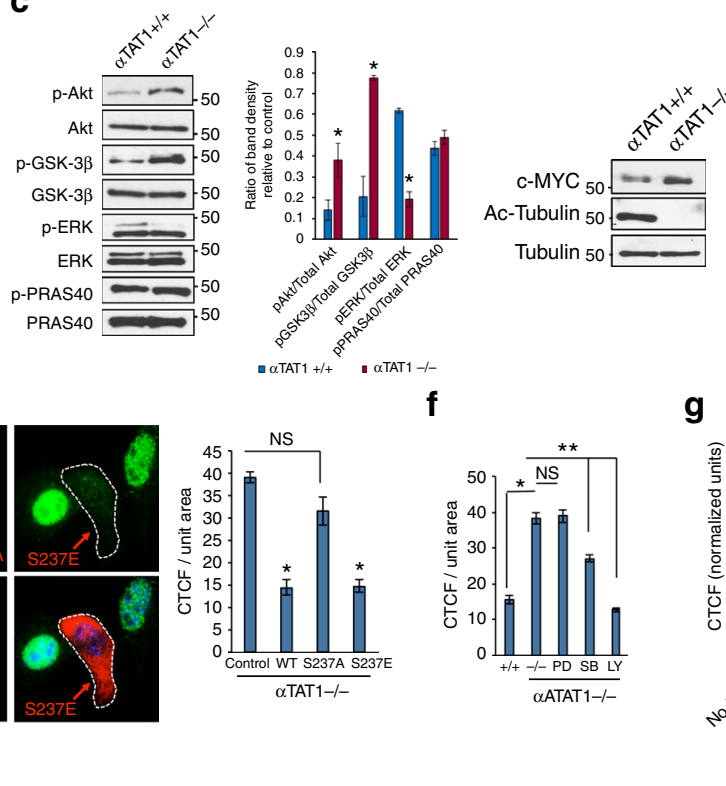

f

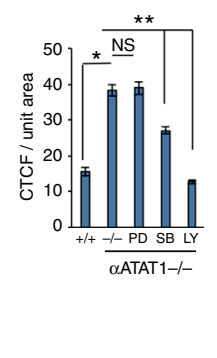

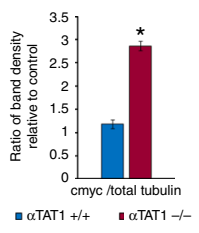

g

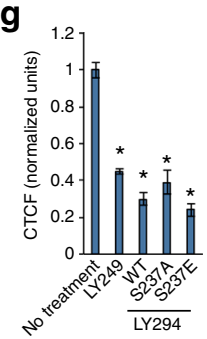

Fig. $5 \alpha$ TAT-Ser237 phosphorylation regulates cell proliferation. a Crystal Violet growth assay of $\alpha \mathrm{TAT} 1^{+/+} \mathrm{MEFs}, \alpha \mathrm{TAT} 1^{-/-}$MEFs, and $\alpha \mathrm{TAT} 1^{-/-}$MEFs rescued with ectopic expression of $\alpha$ TAT1 WT over the period of 24 and $48 \mathrm{~h}$. Presented data are average of four independent experiments. Data were analyzed by two-way ANOVA with Bonferroni post-hoc test: ${ }^{\star} p<0.05$. b Crystal Violet growth assay of $\alpha \mathrm{TAT} 1^{-/-}$MEFs, $\alpha \mathrm{TAT} 1^{-/-}$MEFs rescued with ectopic $\alpha$ TAT1 WT, S237A, or S237E. Presented data is average of three independent experiments. Data were analyzed by two-way ANOVA with Bonferroni post-hoc test: ${ }^{\star} p<0.05$. c Western blot analyses demonstrating levels of pERK, total ERK, pAKT S473, total AKT, c-MYC, pGSK3 $\beta$, total GSK3 $\beta$,

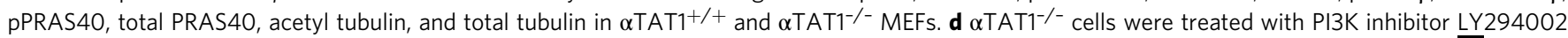
$(20 \mu \mathrm{M})$ or pan AKT inhibitor GSK690693 (10 $\mu \mathrm{M})$ for $1 \mathrm{~h}$, fixed and imaged for c-MYC immunofluorescence staining. CTCF per unit area was quantified using Image J, at least 60 cells were quantified per condition. Error bars represent SEM and type 2 t-test analysis show relative to $\alpha$ TAT1 $/$ - control: ${ }^{\star} p<$ 0.05. e $\alpha \mathrm{TAT1}{ }^{-/-}$cells expressing HA- $\alpha \mathrm{TAT1}$ WT, HA- $\alpha \mathrm{TAT1}$ S237A, or HA- $\alpha \mathrm{TAT1}$ S237E were fixed and imaged for $\alpha \mathrm{HA}$ (red) and c-MYC (green)

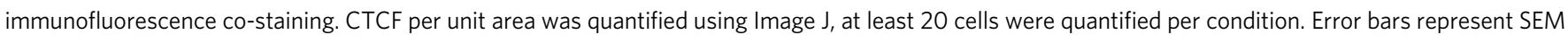
and type 2 t-test analysis show relative to control: ${ }^{\star} p<0.00001, \mathrm{NS}(p=0.3)$. f $\alpha \mathrm{TAT}{ }^{-/-}$cells were treated with ERK1 inhibitor PD98059 (20 $\left.\mu \mathrm{M}\right)$, p38 inhibitor SB203580 (15 $\mu \mathrm{M})$, and PI3K inhibitor LY294002 (20 $\mathrm{MM})$ for 30 min, fixed, and imaged for c-MYC immunofluorescence staining. CTCF per unit area was quantified using Image $J$, at least 60 cells were quantified per condition. Error bars represent SEM and type 2 t-test analysis show relative to $\alpha \mathrm{TAT} 1^{+/+}$control: ${ }^{\star} p<0.05$ and relative to $\alpha \mathrm{TAT} 1^{-/-}$control: ${ }^{\star \star} p<0.05$. $\mathrm{g} \alpha \mathrm{TAT} 1^{-/-}$cells were rescued with ectopic expression of HA- $\alpha \mathrm{TAT} 1 \mathrm{Wt}$, HA$\alpha$ TAT1 S237A or HA- $\alpha$ TAT1 S237E and were treated with LY294002 (20 $\mu$ M) for 30 min before fixing. The conditions were imaged for $\alpha H A$ (red) and cMYC (green) immunofluorescence co-staining. CTCF per unit area was quantified using Image J, at least 30 cells were quantified per condition, normalized with respect to control condition. Error bars represent SEM and type 2 t-test analysis show relative to normalized control: ${ }^{\star} p<0.0001$ 
a

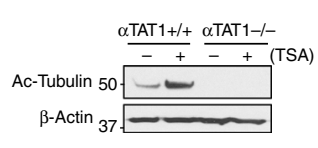

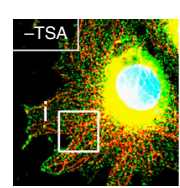
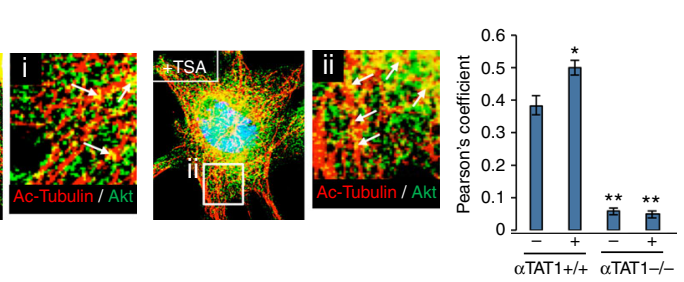

C
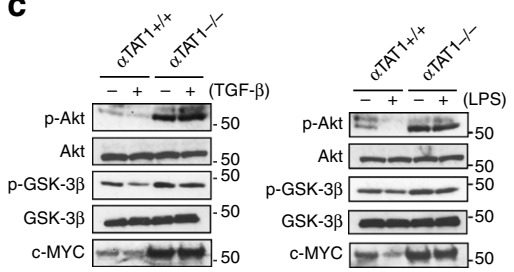

d
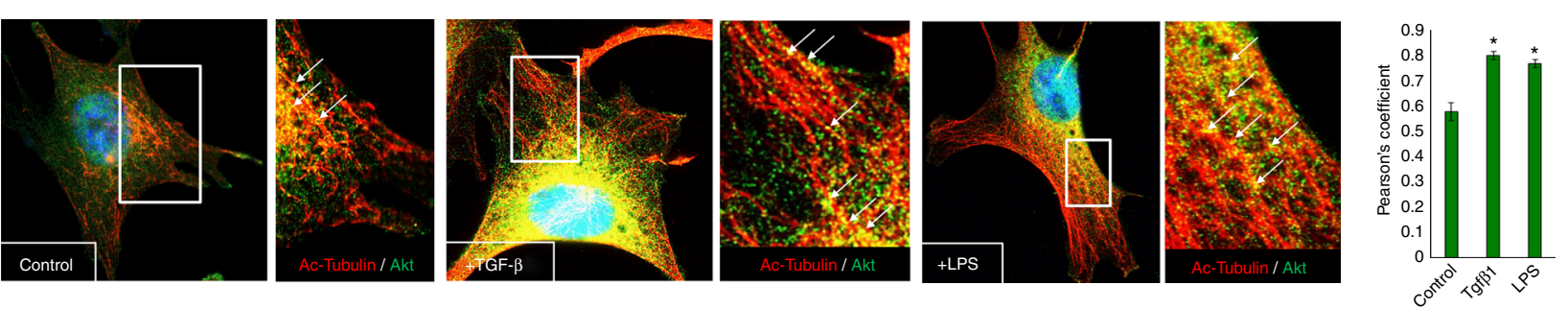

Fig. 6 MT acetylation inhibits AKT activation. a Western blot analyses demonstrating acetyl tubulin level post Trichostatin A (TSA; 500 nM) treatment for 30 min in $\alpha \mathrm{TAT} 1^{+/+}$and $\alpha \mathrm{TAT} 1^{-/-}$MEFs. b $\alpha \mathrm{TAT} 1^{+/+}$and $\alpha \mathrm{TAT} 1^{-/-}$MEFs were treated with $500 \mathrm{nM}$ of $\mathrm{TSA}$ for 30 min before fixing and immunofluorescence staining for acetyl tubulin (red) and total Akt (green). Representative images are obtained from $\alpha \mathrm{TAT} 1^{+/+} \mathrm{MEFs}$ with and without TSA treatment. Acetyl tubulin levels were undetectable in $\alpha T A T 1^{-/-}$cells. Pearson's correlation coefficient was calculated to determine the proximity between acetyl tubulin and total Akt. Image J plugin called Just another co-localization plugin (JACoP) was used to quantify the images. Thirty cells per condition and three ROIs per cell were quantified. Error bars represent SEM and type $2 t$-test analysis show relative to $\alpha \mathrm{TAT} 1^{+/+}$control: ${ }^{\star} p<0.05,{ }^{\star \star} p<$ 0.0001. c $\alpha \mathrm{TAT} 1^{+/+}$and $\alpha \mathrm{TAT} 1^{-/-}$MEFs were treated with TGF- $\beta 1$ (200 pM; $30 \mathrm{~min}$ ) or LPS (10 ng/ml; $30 \mathrm{~min}$ ) before western blot analyses of the

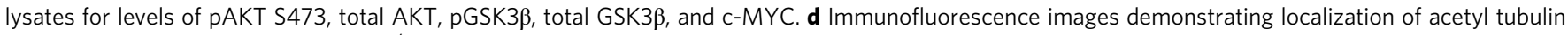
(red) and total Akt (green) in $\alpha \mathrm{TAT} 1^{+/}+$MEFs post treatment with TGF- $\beta 1$ (200 pM; 30 min) or LPS (10 ng/ml; 30 min). Pearson's correlation coefficient was calculated using JACoP, 15 cells per condition, and 3 ROls per cell were quantified. Error bars represent SEM and type $2 t$-test analysis show relative to $\alpha \mathrm{TAT} 1^{+/+}$control: * $p<0.05$

epithelial cell types including HeLa, COS-7, and PANC1 where WT and S237E suppressed cell proliferation over control cells, whereas aTAT1 ${ }^{\mathrm{S} 237 \mathrm{~A}}$ expression had minimal impact even in the presence of TAK1 coexpression (Supplementary Fig. 5A-C).

As we observed minimal changes in the overall MT architecture, we reasoned that acetylation might have a more prominent role in the intracellular transport of mitogenic signaling molecules. Various growth-related signaling pathways therefore were screened in control and aTAT1 knockout cells including ERK, p38, JNK, and AKT along with many other signaling effectors of cell growth and survival (Fig. 5c, graphs). Surprisingly, most growth-promoting pathways remained relatively constant or were only modestly affected, with the exception of p38 and AKT (Fig. 5c and Supplementary Fig. 5D). Although follow-up aTAT1 rescue in aTAT1 ${ }^{-/-}$MEFs ruled out p38 as being independent of cell growth or MT acetylation, AKT activity was distinctly negatively regulated by aTAT1 expression (Fig. 5c). More notably, the enhanced AKT activation in aTAT1-null cells led to the prominent phosphorylation of a key downstream substrate, GSK3 $\beta$, but not PRAS40, another substrate involved in proliferation but is largely insulin-responsive, suggesting that aTAT1 regulation of AKT signaling is highly selective during cell growth suppression (Fig. 5c). Consistent with this notion, we found that GSK3 $\beta$-inactivating phosphorylation promoted cMYC levels, a major driver of cell cycle progression (Fig. 5c). Indeed, previous work has shown that AKT phosphorylation of GSK3 $\beta$ inactivates its kinase activity towards c-MYC at Thr58, a site that promotes $\mathrm{c}-\mathrm{MYC}$ proteasomal degradation upon phosphorylation. To determine whether c-MYC elevation is largely dependent on this pathway, $\alpha \mathrm{TAT} 1^{-/-}$MEFs were treated with an inhibitor of PI3-Kinase (LY294002) or Akt (GSK690693). As observed by immunofluorescence, both PI3-Kinase and Akt inhibition suppressed c-MYC expression relative to control (Fig. 5d). The role of aTAT1 in regulating the c-MYC level was also evident, as reconstituting the expression of aTAT1 WT,
S237A, or S237E in aTAT1 ${ }^{-1-}$ cells showed c-MYC levels being strongly suppressed in the WT and S237E but not S237Aexpressing cells (Fig. 5e and Supplementary Fig. 5E, and graphs). In parallel experiments, we quantified these results upon blocking ERK, p38 and PI3K/AKT activity and found reduced c-MYC levels comparable to WT upon blocking PI3K activation, suggesting that aTAT1 suppresses AKT activation by at least partially inhibiting its recruitment to the membrane (Fig. 5f), a finding that was further substantiated by the fact that both the aTAT $^{-/-}$and S237A expressing cells failed to augment c-MYC expression when the PI3K/AKT pathway was blocked (Fig. $5 \mathrm{~g}$ ).

Many signaling molecules including AKT have been shown to associate with MTs as a mode of intracellular transport. We hypothesized that acetylation sequesters MT-bound AKT, thereby preventing access to the plasma membrane for activation. To test this, control and aTAT1 ${ }^{-/-}$MEFs were first treated with Trichostatin A (TSA), a broad pharmacologic inhibitor of deacetylases, and found a substantial increase in WT MEFs, whereas $a \mathrm{TAT} 1^{-/-}$cells were devoid of tubulin acetylation as expected (Fig. 6a). Similarly, immunofluorescence studies revealed prominent co-localization of acetylated MTs with endogenous AKT at basal state, which was further enhanced upon TSA treatment (Fig. 6b arrows and Supplementary Fig. 6A). Moreover, MT acetylation inversely correlated with AKT activation in which aTAT1 $1^{-/-}$cells, devoid of MT acetylation, produced greater AKT activation irrespective of TSA, whereas WT cells showed diminished activity upon treatment (Supplementary Fig. 6B). More importantly, AKT activation along with GSK $3 \beta$ and c-MYC levels strongly correlated with TAK1 activity, as both TGF- $\beta$ and LPS attenuated these pathways in WT but not in $\alpha \mathrm{TAT} 1^{-/-}$MEFs (Fig. 6c), findings consistent with AKT retention along acetylated MTs to prevent intracellular transport to the plasma membrane for activation. In fact, as assessed by both co-immunoprecipitation and immunofluorescence, this selective association between AKT and acetylated MTs was 
a

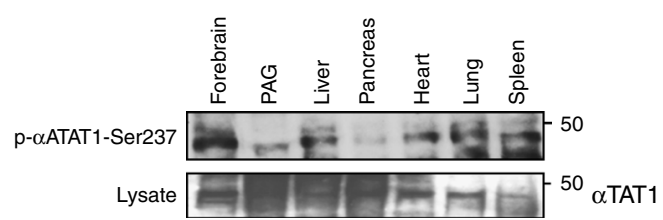

b

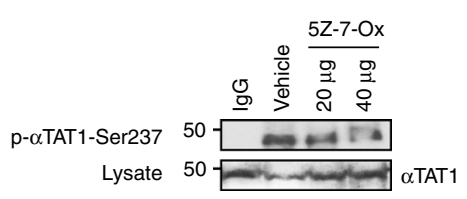

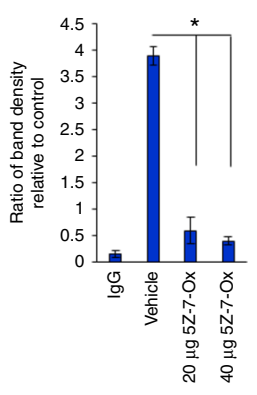

d

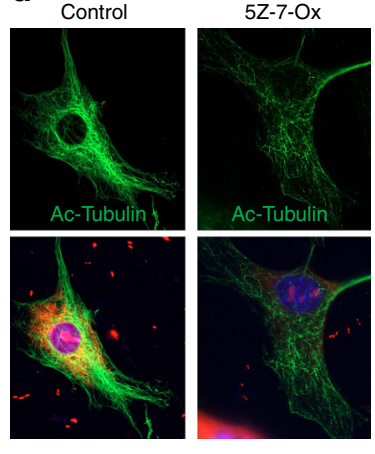

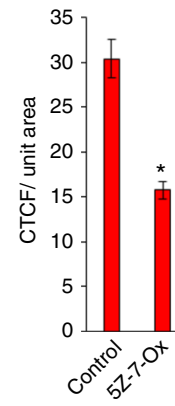

Fig. 7 TAK1-dependent $\boldsymbol{\alpha}$ TAT1 activity in the brain. a Western blot analyses demonstrating phosphorylation levels on serine-237 residue of $\alpha$ TAT1 in lysates extracted from mouse tissues such as forebrain, periaqueductal gray (PAG), liver, pancreas, heart, lung, and spleen. b Representative western blottings demonstrating phospho- $\alpha$ TAT1-Ser237 and total $\alpha$ TAT1 levels in lysates extracted from mice $(n=4)$ with and without treatment with TAK1 inhibitor 5Z-7-oxozeaenol ( $20 \mu \mathrm{g}$ or $40 \mu \mathrm{g}$ per animal; $1 \mathrm{~h}$ ). Band density of four independent blots was calculated using ImageJ. Error bars represent SEM and type $2 t$-test analysis show relative to control: ${ }^{*} p<0.05$. c Representative western blottings demonstrating acetyl tubulin, total tubulin, $\mathrm{pGSK} 3 \beta, \mathrm{cMYC}$ and PAKT-S473 levels in lysates extracted from mice $(n=4)$ with and without treatment with TAK1 inhibitor 5Z-7-oxozeaenol (20 $\mu$ g or $40 \mu \mathrm{g}$ per animal; $1 \mathrm{~h})$. Band density of four independent blots was calculated using ImageJ. Error bars represent SEM and type $2 t$-test analysis show relative to control: ${ }^{\star} p<$ 0.05 (pAKT), ${ }^{\star \star} p<0.05$ (cMYC), ${ }^{\star \star \star} p<0.05$ (acetyl tubulin), NS is not statistically significant (pGSK3 $\beta$ ). d Immunofluorescence images demonstrating acetyl tubulin (green) levels in neurons (red, Nissl stain) extracted from the dorsal root ganglia (DRG) of mice. The neurons were subjected to TAK1 inhibitor 5Z-7-oxozeaenol ( $20 \mu \mathrm{M} ; 1 \mathrm{~h}), 24 \mathrm{~h}$ post dissection. CTCF per unit area was quantified using Image J, at least 40 cells were quantified per condition. Error bars represent SEM and type $2 t$-test analysis show relative to control: ${ }^{\star} p<0.05$

greatly enhanced in WT but not $\alpha \mathrm{TAT} 1^{-/-}$MEFs upon treatment with TAK1 inducers (i.e., TGF- $\beta$ and LPS) and even paclitaxel, an inhibitor of MT depolymerization that typically causes MT acetylation (Fig. 6d and Supplementary Fig. 6C).

Finally, in order to gain novel functional insights in vivo, we screened for relative aTAT1 catalytic activity in mouse using our novel phospho-Ser237 antibody and found that large portions of the brain contained some of the highest levels among the various tissue types (Fig. 7a). Here, TAK1 was inhibited through intracerebroventricular injection of 5Z-7-Ox and subsequently analyzed for p-aTAT1-Ser237 levels. There was a dose-dependent decrease in phosphorylation (Fig. 7b) as predicted, although more strikingly, MT acetylation was dramatically reduced while pAKT, p-GSK3 $\beta$, and c-MYC levels all increased (Fig. 7c, graph). Similarly, freshly isolated dorsal root ganglion (DRG) cells briefly exposed to 5Z-7-Ox also displayed significantly diminished MT acetylation (Fig. 7d). As illustrated in Supplementary Fig. 7, these combined in vitro and in vivo results provide compelling evidence for the role of TAK1 in aTAT1 regulation of GSK3 $\beta$ and c-MYC signaling through MT-based AKT sequestration and inactivation.

\section{Discussion}

As canonical mediators of TGF- $\beta$ signaling, receptor-activated SMADs produce pleiotropic effects by shuttling to the nucleus to regulate the expression of hundreds of target genes in a highly cell-type and context-specific manner ${ }^{13,27}$. This requires SMAD proteins to associate with various kinesin and dynein motor proteins to move along the microtubular network ${ }^{28,29}$. In the present study, we report on what is believed to be the first nontranscriptional regulation of MT functions by TGF- $\beta$ signaling mediated by rapid TAK1 activation of aTAT1. These findings have broad and significant pathophysiologic implications as aberrant TGF- $\beta$ signaling through TAK1 underlies numerous conditions including cancer, inflammatory and neurologic disorders. Although beyond the scope of the present study, future exploration of how TGF- $\beta$-related changes in aTAT1 function and MT acetylation contribute to these cellular and disease states will be vitally important as new TGF- $\beta$-targeted therapies continue to emerge in clinical settings.

Very recently, TGF- $\beta$ has been shown to modulate MT acetylation during epithelial-mesenchymal transition ${ }^{30,31}$. Although the mechanisms are still being investigated, they appear to be based on context-specific transcriptional events, as acetylation reportedly occurs in the course of $48 \mathrm{~h}$ in epithelial cells. Our central findings represent a broader scale as we observed TAK1induced MT acetylation in response to TGF- $\beta 1$ that persists for at least $2 \mathrm{~h}$ across multiple cell types including mouse fibroblasts, human epithelial, and endothelial cells. It is also noteworthy that similar effects were observed upon stimulation with LPS, another potent inducer of TAK1 tied to inflammatory process.

The precise role of tubulin acetylation in MT stability has long remained controversial, as various genetic, cellular, and in vitro findings have ranged from only minor effects to being required for the overall MT integrity ${ }^{4}$. However, most recent data provide 
compelling new evidence in support of structural stability, as acetylation appears to protect long-lived MTs against mechanical ageing and resistance to mechanical breakage ${ }^{32,33}$. Although our work did not specifically examine these aspects, we found no discernable signs of breakage or depolymerization of existing MTs upon depletion or inhibition of TAK1 activity, nor in response to the overexpression of the various aTAT1 constructs. This finding surprisingly contrasts with at least one report in which aTAT1 overexpression was shown to destabilize MTs through its interaction rather than its acetyltransferase activity ${ }^{34}$. Instead, our data support the primary role of TAK1 in regulating aTAT1 enzymatic activity rather than its recruitment to or physical association with MTs.

Over the years, numerous cellular stresses have been shown to induce MT acetylation including UV irradiation, osmotic, immune, and viral responses. Hence, it is not surprising that aTAT1 is also modulated by various stress stimuli, although in most cases the underlying mechanisms remain poorly defined. In fact, with the exception of one previous study examining the role of ROS-mediated AMPK activation, little is known about how aTAT1 is regulated. In this study, ROS-mediated AMPK activation reportedly enhanced aTAT1 activity through phosphorylation, though whether aTAT1 is a direct AMPK phosphosubstrate remains uncertain ${ }^{12}$.

Interestingly, given that TAK1 can activate AMPK in many contexts, it remains to be determined whether the ROS-AMPK pathway controlling aTAT1 requires TAK1 activity. Nevertheless, it seems likely to be that TAK1 regulation of aTAT1 can occur independently of AMPK, as our work demonstrates that the TAK1/aTAT1 interaction is direct and that Ser237 phosphorylation occurs even in an in vitro setting using purified proteins. Still, considering the fact that TAK1 is activated by such diverse stress stimuli across cell types, it will be important to fully characterize the relationship between TAK1 and AMPK activity in regulating aTAT1 and MT acetylation.

MTs have a key role in intracellular transport of protein cargo through kinesin-1 and kinesin-like motor proteins, which preferentially bind to stable MTs marked by acetylation and detyrosination $^{5}$. In our studies, genetic and pharmacologic manipulation of tubulin acetylation revealed that MT acetylation has a strong sequestering effect rather than dynamic transport of AKT. Based on our data, it is likely to be that AKT sequestration is at least partially selective, as mitogenic and other cell growthrelated pathways seem largely unaffected by MT acetylation.

A notable observation was that, contrary to our expectation, there were no aTAT1 or MT acetylation-dependent changes in TGF- $\beta$-responsive Smad 2/3 activation even though it is a potent inhibitor of cell growth that also requires MT-based transport. Taken together, our findings support a working model in which TGF- $\beta$-responsive TAK1 phosphorylates and activates $\alpha \mathrm{TAT} 1$ to enhance MT acetylation, which in turn, preferentially binds to and sequesters $\mathrm{AKT}$ to prevent its access to the plasma membrane where it normally becomes activated.

Although our in vivo findings demonstrate that TAK1 activation of aTAT1 and its downstream impact on AKT signaling goes beyond cultured cell lines, the pathophysiologic outcome of altering TAK1 activity particularly in the brain may prove much more complex and warrants further investigation. Indeed, given that aTAT1-null mice are viable yet exhibit cognitive dysfunctions, it is possible that only certain regions of the brain or neuronal subtypes that depend more highly on TAK1 activity are susceptible to neurodegenerative dysfunction.

In summary, the present work establishes a fundamental new role for TGF- $\beta$ in regulating MT functional dynamics. Activation of TAK1 by TGF- $\beta$ superfamily ligands as well as LPS can cause phosphorylation-induced aTAT1 activation and MT acetylation.
Our findings reveal novel pathophysiologic implications between TGF- $\beta$ signaling and aberrant MT acetylation in cancer and neurodegenerative disorders.

\section{Methods}

Antibodies. The following antibodies used in this study were purchased from Cell Signaling: acetyl- $\alpha$-tubulin (1:1000; \#5335), TAB1 (1:1000; \#3226), pERK (1:2000; \#9101), total ERK (1:2000; \#4695), pAKT-S473 (1:1000; \#4060), pP38 (1:2000; \#4511), pJNK (1:2000; \#9255), cMYC (1:2000; \#13987), pGSK33-Ser9 (1:2000; \#5558), total GSK3 3 (1:2000; \#12456), pTAK1 (1:1000; \#9339), total TAK1 (1:1000; \#4505), total AKT (1:1000; \#4691), pPRAS40 (1:2000; \#2997), total PRAS40 (1:2000; \#2610), pSmad2 (1:1000; \#8828), and Myc-Tag (1:4000; \#2276). Total tubulin YOL1/34 (1:2000; MCA78G) (Abcam), aHA (1:3000; \#11867431001), $\alpha$ Flag (1:4000; F1804) (Sigma), aTAT1 phosphoserine 237 (1:250) (Thermo Scientific), and aTAT1 (1:500; sc-167354) (Santa Cruz) were also used.

Animal studies. Male CD-1 (ICR) mice in age-matched controlled cohorts from 4 to 8 weeks of age were used for all experiments and were obtained from Charles River Laboratories (Wilmington, MA). Mice were kept in an animal facility at University of Arizona, no more than five mice per cage, under environmental temperature and humidity control with $12 \mathrm{~h}$ light/dark cycles and free access to food and water. All experiments performed were in accordance with Institutional Animal Care and Use Committee-approved protocols of the University of Arizona. For the dose-response of TAK1 inhibitor 5Z-7-oxozeaenol experiments, the mice were injected I.C.V. with 20 or $40 \mu \mathrm{g}$ of 5Z-7-Ox or vehicle into left ventricle of the brain for $1 \mathrm{~h}$ before killing by rapid cervical dislocation. The brain was removed and rapidly frozen on liquid nitrogen before use. For DRG isolation, the spines from freshly sacrificed mice were dissected out and DRGs were extracted from the spinal cord and stored in the DRG+ media (Dulbecco's modified Eagle's medium (DMEM), fetal bovine serum (FBS), and Normocin) before incubation in collagenase/protease mixture for $1 \mathrm{~h}$ at $37^{\circ} \mathrm{C}$. Post-incubation DRGs were washed and reincubated in DRG + media containing NGF ( $30 \mathrm{ng} / \mu \mathrm{l})$. The DRGs were plated on poly-D-lysine-coated coverslips for immunofluorescence studies.

Plasmid, constructs, and shRNAs. The GFP- $\alpha$ TAT construct was obtained from Addgene (Plasmid \#27099; Dr. Maxence Nachury) and Flag-TAK1, Flag-TAK1$\mathrm{K} 63 \mathrm{~W}$, and TAB1 constructs were generous gifts from Dr. Jianhua Yang (Baylor College of Medicine). The Flag-Tak1 $\Delta 300$ mutant was generated by PCR amplification using Flag-TAK1-WT as a template and forward and reverse primer complementary to the sequences near the defined regions. The HA- $\alpha \mathrm{TAT}$ construct was generated by PCR amplification using GFP-aTAT as a template. HAaTAT $\Delta 196$ and $\Delta 301$ contstructs were generated by using HA- $\alpha$ TAT-WT as template with forward and reverse primers complementary to the sequences near the defined regions. HA- $\alpha$ TAT point mutations (S200A, T204A, S207A, T215A, S236A, S237A, T261A, S272A, S276A, S315A) were generated by site directed mutagenesis using HA- $\alpha$ TAT-WT as template and specific forward primer for each and reverse primer. TAK1 stable knockdown cells were generated in Hela cells was achieved by transfecting cells separately with two shRNA vector purchased from sigma (Mission shRNA \# TRCN0000001556, (5'-CCGGCAGTGTGTCTTGTGAT GGAATCTCGAGATTCCATCACAAGACACACTGTTTTT-3') and

\# TRCN0000001557, (5'-CCGGGACACACATGACCAATAACAACTCGAG GTTGTTATTGGTCATGTGTGTCTTTTT- ${ }^{\prime}$ )

Transfected cells were selected in media containing $3 \mathrm{mg} / \mathrm{mL}$ puromycin, and stable colonies with Smad2 depletion were confirmed by western blotting.

Cell culture and transfection. COS-7, HeLa, and MEFs were cultured in DMEM (GIBCO) supplemented with 10\% FBS (HyClone). Mouse embryonic endothelial cells were maintained in MCDB-131 (GIBCO) supplemented with $10 \%$ FBS, $2 \mathrm{mM}$ L-glutamine, endothelial cell growth supplement (Sigma). aTAT $1^{-/-}$MEFs were a generous gift from Dr. Maxence Nachury (Stanford). HeLa sh-TAK1 knockdown stables were generated first by small hairpin RNA vector transfection, selected in puromycin $(1-2 \mathrm{mg} / \mathrm{mL})$, then colonies were isolated and biochemically validated for TAK1 knockdown. Transfection was performed using Lipofectamine 2000 (Invitrogen).

Immunoprecipitation. Cells were washed with phosphate-buffered saline (PBS), lysed on ice with lysis buffer for $20 \mathrm{~min}(20 \mathrm{mM}$ HEPES pH 7.4, $150 \mathrm{mM} \mathrm{NaCl}, 5$ $\mathrm{mM} \mathrm{NaF}, 1 \% \mathrm{NP}-40$ ) before centrifugation at 13,000 r.p.m. for $13 \mathrm{~min}$. Supernatants were incubated with appropriate antibodies and agarose $\mathrm{G}$ or protein $\mathrm{A}$ agarose for $6 \mathrm{~h}$ at $4 \mathrm{C}$. Immunoprecipitates were then pelleted and washed three times before storing them in $2 \times$ sample buffer followed by western blot analyses.

Immunofluorescence studies. Cells grown on gelatin or poly-L-lysine (GIBCO) coated coverslips were fixed with $4 \%$ paraformaldehyde, permeabilized in $0.1 \%$ Triton X-100 in PBS for 4 min, then blocked with 5\% bovine serum albumin in PBS for $20 \mathrm{~min}$. The primary antibody and fluorescently conjugated secondary antibodies were incubated at room temperature for $1.5 \mathrm{~h}$. The cover slips were 
mounted using anti-fade that contained 4',6-diamidino-2-phenylindole. Confocal Imaging was performed on Nikon A1R laser microscope.

Crystal violet growth assay. $\alpha \mathrm{TAT} 1^{+/+}$or $\alpha \mathrm{TAT} 1^{-/-}$was plated at a density of 10,000 cells per well in 12-well plates. HeLa scrambled control or HeLa shTAK1 were plated at a density of 15,000 cells per well in 12-well plates. Conditions involving ectopic expression of aTAT1 or TAK1 constructs were fixed (4\% paraformaldehyde for $17 \mathrm{~min}$ ) at different time points starting at $24 \mathrm{~h}$ post transfection. Following fixation, cells were washed with $1 \times$ water and stained with $0.1 \%$ crystal violet for $30 \mathrm{~min}$. Cells were washed with $1 \times$ miili $\mathrm{Q}$ water repeatedly and air dried for $30 \mathrm{~min}$. Cells were de-stained using crystal violet de-staining solution ( $10 \%$ acetic acid, $50 \%$ methanol, $40 \% \mathrm{H}_{2} \mathrm{O}$ ) for $15 \mathrm{~min}$ and the optical density was read at $590 \mathrm{~nm}$ in a microplate reader.

Cloning, generation of recombinant baculoviruses, and transfection. Genes coding for full-length aTAT, TAK1, and TAB1 were cloned in a pFastBac ${ }^{\text {tw }}$-Dual vector (Life Technologies). aTAT was cloned between the EcoR1 and Hind III restriction site from a DNA fragment produced by PCR using the WT gene as template, amplified with $5^{\prime}$ - and $3^{\prime}$-hexahistidine-tagged primer (5'-GC GAA TTC GCC ATG GGT ATG CAC CAC CAC CAC CAC CAC GAG TTC CCG TTC GAT GTG-3' and 5'-CCC AAG CTT TTA GTA TCG ACT CTC CTC-3'). TAK1 was cloned between EcoR1 and Kpn1 restriction site from a DNA fragment produced by PCR using the WT gene as template, amplified with $5^{\prime}$ - and $33^{\prime}$-hexahistidine-tagged primer ( $5^{\prime}$-GC GAA TTC GCC ATG GGT ATG CAC CAC CAC CAC CAC CAC TCT ACA GCC TCT GCC GCC-3' and 5'-CGG GGT ACC TCA TGA AGT GCC TTG TCG-3' ${ }^{\prime}$ ). TAB1 was cloned between the EcoR1 and Hind III restriction site from a DNA fragment produced by PCR using the WT gene as template, amplified with $5^{\prime}$ - and $3^{\prime}$-hexahistidine-tagged primer $\left(5^{\prime}\right.$-GC GAA TTC GCC ATG GGT ATG CAC CAC CAC CAC CAC CAC GCG GCG CAG AGG AGG AGC-3' and 5'-CCC AAG CTT CTA CGG TGC TGT CAC CAC-3'). Once constructed, each plasmid was checked by sequencing and colony-PCR and used to transform MAX Efficiency ${ }^{\circledR}$ DH10Bac ${ }^{\mathrm{TM}}$ competent cells for production of recombinant bacmids by using Bac-to-Bac ${ }^{\circledR}$ Baculovirus Expression System. Transfection was performed in SF21 insect cell line by using Cellfectin II reagent (Invitrogen) according to protocols provided by manufacturer. Purified recombinant viruses were amplified with three rounds of infection in Sf 21 cells grown at $27^{\circ} \mathrm{C}$ using a multiplicity of infection (MOI) of 0.1. Viral supernatants were harvested $48-72 \mathrm{~h}$ post infection.

Purification of recombinant proteins. Sf 21 cells were infected with baculovirus at an MOI of 2 . After $48 \mathrm{~h}$ post infection, cells were collected by centrifugation at $1000 \times g$ for $3 \mathrm{~min}$, gently washed with resuspension buffer $(20 \mathrm{mM}$ HEPES at $\mathrm{pH}$ $7.4,0.5 \mathrm{M} \mathrm{NaCl}, 250 \mathrm{mM}$ sucrose, a protease inhibitors $[5 \mu \mathrm{g} / \mathrm{ml}$ aprotinin, $5 \mu \mathrm{g} / \mathrm{ml}$ leupeptin, $2 \mu \mathrm{M}$ pepstatin A, $1 \mathrm{mM}$ PMSF]). Cells were again plated and then resuspended with sucrose free resuspension buffer (minus sucrose) and then lysed using a sonication (Vibro Sonics) at $50 \%$ amplitude with $5 \mathrm{~s}$ on and $5 \mathrm{~s}$ off for 10 min. Sonication and all subsequent steps were performed in $4{ }^{\circ} \mathrm{C}$ or on ice. After sonication, the cell lysate was centrifuged at 11,000 r.p.m. for $20 \mathrm{~min}$ and the supernatant was collected and supplemented with $0.05 \%$ Triton X-100. The cellfree supernatant was loaded onto HisPur cobalt resin column (Thermo Scientific) pre-equilibrated with resuspension buffer $(10 \mathrm{ml})$. The column was washed first with $30 \mathrm{ml}$ of buffer (20 mM HEPES ( $\mathrm{pH} 7.4), 0.5 \mathrm{M} \mathrm{NaCl}, 12 \mathrm{mM}$ imidazole, and $0.05 \%$ Triton X-100), then washed with additional 10 volumes of TritonX-100-free wash buffer. Recombinant Hexahistidine-tagged proteins were eluted with buffer containing $20 \mathrm{mM}$ HEPES (pH 7.4), $0.5 \mathrm{M} \mathrm{NaCl}, 150 \mathrm{mM}$ Immidazole (20 ml), and $0.5 \mathrm{ml}$ fractions were collected. Protein fractions were resolved on $10 \%$ SDSpolyacrylamide gel electrophoresis (PAGE). The fractions containing purified protein were pooled and dialyzed extensively against 20 mM HEPES (pH 7.4) 100 $\mathrm{mM} \mathrm{NaCl}$ at $4{ }^{\circ} \mathrm{C}^{35}$

Nocodazole-based kinetics. COS-7 cells expressing HA- TAT1 WT or HAaTAT1-S237A were subjected to nocodazole $(100 \mathrm{nM})$ for $1 \mathrm{~h}$, washed thoroughly with PBS, and then immediately fixed after $0,15,30,45,60,90$, and $120 \mathrm{~min}$. The fixed cells were then imaged for aHA and Acetyl tubulin immunofluorescence costaining. The images were quantified by skeletonizing the acetyl tubulin staining using Image J and skeletons analyzed for length and branching of acetyl tubulin filaments.

In vitro kinase assay. Kinase assays were typically performed at $25^{\circ} \mathrm{C}$ by preincubating a $0.5 \mu \mathrm{M}$ concentration of protein in $1 \mathrm{mM} \mathrm{MnCl} 2$ for $5 \mathrm{~min}$ in buffer (20 mM HEPES at pH 7.4, $50 \mathrm{mM} \mathrm{NaCl}$ ) and then initiated by addition of $100 \mu \mathrm{M}$ ATP. The reactions were stopped after the noted period $(30 \mathrm{~min})$ then did immunoprecipitation with pS237A (custom antibody Thermo Scientific). Proteins were resolved by SDS-PAGE and immunoblot with Total S237A (custom antibody Thermo Scientific), with enhanced chemiluminescence (ECL Thermo Scientific) ${ }^{35}$.

In vitro kinase and acetylation reaction method. In vitro kinase assays were by preincubating purified TAK1/TAB1 and the kinase substrate aTAT1 $(0.5 \mu \mathrm{M}$ each) in $1 \mathrm{mM} \mathrm{MnCl}$ for $5 \mathrm{~min}$ in buffer (20 mM HEPES at pH 7.4, $50 \mathrm{mM} \mathrm{NaCl})$, then initiating the reaction by addition of $100 \mu \mathrm{M}$ ATP. The reactions were stopped after $30 \mathrm{~min}$. An aliquot of the kinase reaction was subsequently transferred to perform an in vitro acetylation reaction, carried out in $10 \mu \mathrm{l}$ ADE buffer $(40 \mathrm{mM}$ Pipes pH 6.9, $0.8 \mathrm{mM}$ EGTA, $0.5 \mathrm{mM} \mathrm{MgSO}_{4}, 1 \mathrm{mM}$ dithiothreitol) with purified bovine brain tubulin (1 $\mu \mathrm{g}$; Cytoskeleton), phosphorylated aTAT1-WT and aTAT1Ser237A, and $8 \mu \mathrm{M}$ acetyl coenzyme A (Sigma). Reaction mixtures were incubated at $37^{\circ} \mathrm{C}$ for $30 \mathrm{~min}$ and stopped by addition of SDS loading buffer. Proteins were resolved by SDS-PAGE and immunoblot for $\alpha$-tubulin acetylation.

Statistics. Statistical analysis was performed using both student $\mathrm{t}$ test and one-way analysis of variance (ANOVA). Data are presented as mean \pm SEM. For crystal violet proliferation, data analysis was performed using Sigma plot, two-way ANOVA analysis/Bonferroni post-hoc analysis.

Data availability. The authors confim that all data supporting the findings of this study are available within the paper and supplementary data.

Received: 7 August 2017 Accepted: 4 April 2018

Published online: 27 April 2018

\section{References}

1. Desai, A. \& Mitchison, T. J. Microtubule polymerization dynamics. Annu. Rev. Cell. Dev. Biol. 13, 83-117 (1997).

2. Nogales, E. Structural insights into microtubule function. Annu. Rev. Biochem. 69, 277-302 (2000).

3. Subramanian, R. \& Kapoor, T. M. Building complexity: insights into selforganized assembly of microtubule-based architectures. Dev. Cell. 23, 874-885 (2012).

4. Li, L. \& Yang, X. J. Tubulin acetylation: responsible enzymes, biological functions and human diseases. Cell. Mol. Life. Sci. 72, 4237-4255 (2015).

5. Song, Y. \& Brady, S. T. Post-translational modifications of tubulin: pathways to functional diversity of microtubules. Trends Cell. Biol. 25, 125-136 (2015).

6. Kalebic, N. et al. alphaTAT1 is the major alpha-tubulin acetyltransferase in mice. Nat. Commun. 4, 1962 (2013).

7. Kim, G. W., Li, L., Gorbani, M., You, L. \& Yang, X. J. Mice lacking alpha tubulin acetyltransferase 1 are viable but display alpha-tubulin acetylation deficiency and dentate gyrus distortion. J. Biol. Chem. 288, 20334-20350 (2013)

8. Morley, S. J. et al. Acetylated tubulin is essential for touch sensation in mice. Elife 5, e20813 (2016).

9. Montagnac, G. et al. alphaTAT1 catalyses microtubule acetylation at clathrincoated pits. Nature 502, 567-570 (2013).

10. Shida, T., Cueva, J. G., Xu, Z., Goodman, M. B. \& Nachury, M. V. The major alpha-tubulin K40 acetyltransferase alphaTAT1 promotes rapid ciliogenesis and efficient mechanosensation. Proc. Natl Acad. Sci. USA 107, 21517-21522 (2010).

11. Castro-Castro, A., Janke, C., Montagnac, G., Paul-Gilloteaux, P. \& Chavrier, P. ATAT1/MEC-17 acetyltransferase and HDAC6 deacetylase control a balance of acetylation of alpha-tubulin and cortactin and regulate MT1-MMP trafficking and breast tumor cell invasion. Eur. J. Cell. Biol. 91, 950-960 (2012).

12. Mackeh, R. et al. Reactive oxygen species, AMP-activated protein kinase, and the transcription cofactor p300 regulate alpha-tubulin acetyltransferase-1 (alphaTAT-1/MEC-17)-dependent microtubule hyperacetylation during cell stress. J. Biol. Chem. 289, 11816-11828 (2014).

13. Derynck, R. \& Zhang, Y. E. Smad-dependent and Smad-independent pathways in TGF-beta family signalling. Nature 425, 577-584 (2003).

14. Massague, J. TGFbeta signalling in context. Nat. Rev. Mol. Cell. Biol. 13, 616-630 (2012).

15. Massague, J. \& Chen, Y. G. Controlling TGF-beta signaling. Genes Dev. 14, 627-644 (2000).

16. Massague, J., Seoane, J. \& Wotton, D. Smad transcription factors. Genes Dev 19, 2783-2810 (2005).

17. Ninomiya-Tsuji, J. et al. The kinase TAK1 can activate the NIK-I kappaB as well as the MAP kinase cascade in the IL-1 signalling pathway. Nature 398, 252-256 (1999).

18. Morioka, S. et al. TAK1 kinase signaling regulates embryonic angiogenesis by modulating endothelial cell survival and migration. Blood 120, 3846-3857 (2012).

19. Sorrentino, A. et al. The type I TGF-beta receptor engages TRAF6 to activate TAK1 in a receptor kinase-independent manner. Nat. Cell Biol. 10, 1199-1207 (2008).

20. Shibuya, H. et al. TAB1: an activator of the TAK1 MAPKKK in TGF-beta signal transduction. Science 272, 1179-1182 (1996). 
21. Mihaly, S. R., Morioka, S., Ninomiya-Tsuji, J. \& Takaesu, G. Activated macrophage survival is coordinated by TAK1 binding proteins. PLoS ONE 9, e94982 (2014)

22. Mihaly, S. R., Ninomiya-Tsuji, J. \& Morioka, S. TAK1 control of cell death Cell Death Differ. 21, 1667-1676 (2014).

23. Mao, R. et al. TAK1 lysine 158 is required for TGF-beta-induced TRAF6mediated Smad-independent IKK/NF-kappaB and JNK/AP-1 activation. Cell. Signal. 23, 222-227 (2011).

24. Tavares, A. L., Mercado-Pimentel, M. E. \& Runyan, R. B. \& Kitten, G. T. TGF beta-mediated RhoA expression is necessary for epithelial-mesenchymal transition in the embryonic chick heart. Dev. Dyn. 235, 1589-1598 (2006).

25. Kardassis, D., Murphy, C., Fotsis, T., Moustakas, A. \& Stournaras, C. Control of transforming growth factor beta signal transduction by small GTPases. Febs. J. 276, 2947-2965 (2009).

26. Aguilar, A. et al. Alpha-tubulin K40 acetylation is required for contact inhibition of proliferation and cell-substrate adhesion. Mol. Biol. Cell 25, 1854-1866 (2014).

27. Ehrlich, M., Horbelt, D., Marom, B., Knaus, P. \& Henis, Y. I. Homomeric and heteromeric complexes among TGF-beta and BMP receptors and their roles in signaling. Cell. Signal. 23, 1424-1432 (2011).

28. Schmierer, B. \& Hill, C. S. TGFbeta-SMAD signal transduction: molecular specificity and functional flexibility. Nat. Rev. Mol. Cell. Biol. 8, 970-982 (2007).

29. Moustakas, A. \& Heldin, C. H. Dynamic control of TGF-beta signaling and its links to the cytoskeleton. FEBS Lett. 582, 2051-2065 (2008).

30. $\mathrm{Gu}, \mathrm{S}$. et al. Loss of alpha-tubulin acetylation is associated with TGF-betainduced epithelial-mesenchymal transition. J. Biol. Chem. 291, 5396-5405 (2016).

31. Wu, J. et al. RCCD1 depletion attenuates TGF-beta-induced EMT and cell migration by stabilizing cytoskeletal microtubules in NSCLC cells. Cancer Lett. 400, 18-29 (2017)

32. Portran, D., Schaedel, L., Xu, Z., Thery, M. \& Nachury, M. V. Tubulin acetylation protects long-lived microtubules against mechanical ageing. Nat. Cell. Biol. 19, 391-398 (2017).

33. $\mathrm{Xu}, \mathrm{Z}$. et al. Microtubules acquire resistance from mechanical breakage through intralumenal acetylation. Science 356, 328-332 (2017).

34. Kalebic, N. et al. Tubulin acetyltransferase alphaTAT1 destabilizes microtubules independently of its acetylation activity. Mol. Cell Biol. 33, 1114-1123 (2013).

35. Lee N. Y., Hazlett T. L. \& Koland J. G. Structure and dynamics of the EGF receptor C-terminal phosphorylation domain. Protein Sci. 15, 1142-1152 (2006)

\section{Acknowledgements}

We thank Dr. Jianhuan Yang (Baylor College of Medicine) for the TAK1 constructs and Dr. Maxence Nachury (Stanford) for $\alpha_{\mathrm{TAT}}{ }^{-/-}$MEFs. We acknowledge the Mass Spectrometry and Proteomics Facility at The Ohio State University for their technical assistance. This work was supported in part by NIH CA178443 awarded to N.Y.L., and University of Arizona Cancer Center and University of Arizona Department of Pharmacology, and Department of Chemistry and Biochemistry for internal funding.

\section{Author contributions}

N.Y.L. conceived and designed the study. N.S., S.K., J.C.B., N.Z., C.C.P., and W.L. conducted the experiments. J.M.S., N.H., and K.M., helped design the experiments and reviewed the manuscript. N.S. and N.Y.L. wrote the manuscript.

\section{Additional information}

Supplementary Information accompanies this paper at https://doi.org/10.1038/s41467018-04121-y.

Competing interests: The authors declare no competing interests.

Reprints and permission information is available online at http://npg.nature.com/ reprintsandpermissions/

Publisher's note: Springer Nature remains neutral with regard to jurisdictional claims in published maps and institutional affiliations.

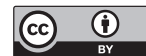

Open Access This article is licensed under a Creative Commons Attribution 4.0 International License, which permits use, sharing, adaptation, distribution and reproduction in any medium or format, as long as you give appropriate credit to the original author(s) and the source, provide a link to the Creative Commons license, and indicate if changes were made. The images or other third party material in this article are included in the article's Creative Commons license, unless indicated otherwise in a credit line to the material. If material is not included in the article's Creative Commons license and your intended use is not permitted by statutory regulation or exceeds the permitted use, you will need to obtain permission directly from the copyright holder. To view a copy of this license, visit http://creativecommons.org/ licenses/by/4.0/

(C) The Author(s) 2018 